\title{
Tarifpolitische Chancen nutzen - Rückkehr zur produktivitätsorientierten Lohnpolitik geboten
}

\author{
Trevor Evans, Arne Heise, Hansjörg Herr, \\ Jürgen Kromphardt, Jan Priewe, Claus Thomasberger
}

\begin{abstract}
Selten waren die Bedingungen für eine Tarifrunde in der Metallindustrie so günstig für die Arbeitnehmerinnen und Arbeitnehmer wie in diesem Jahr, selten weisen alle Daten in gleicher Richtung darauf hin, dass jetzt ein Ende der Politik der Lohnzurückhaltung und falschen Bescheidenheit gesamtwirtschaftlich vernünftig und überdies möglich ist. Notwendig ist eine Rückkehr zu einer mittelfristig ausgerichteten produktivitätsorientierten Lohnpolitik. Dies ist nicht nur ein Schritt zu mehr Verteilungsgerechtigkeit, sondern vor allem gesamtwirtschaftlich geboten: Es wäre gut für Wachstum und Beschäftigung und mit Preisniveaustabilität vereinbar. Um nicht missverstanden zu werden: Wir plädieren nicht für einen einmaligen „kräftigen Schluck aus der Pulle", weil die Konjunktur gerade mal gut läuft, sondern wir halten die Lage für günstig, jetzt auf eine andere Konzeption der Lohnpolitik zu setzen. Wenn in diesem Jahr in der Metallindustrie ein Effektivlohnanstieg deutlich oberhalb von $4,0 \%$ erreicht wird, halten wir dies für vertretbar und vernünftig. Wir begründen dies wie folgt:
\end{abstract}

\section{GESPALTENE KONJUNKTUR BEI SINKENDEN REALLÖHNEN}

Die Konjunktur lief mit 2,5 \% Wirtschaftswachstum 2006 so gut wie seit dem Jahr 2000 nicht, aber sie ist beim privaten Konsum noch nicht angekommen. Immer noch weist die gesamtwirtschaftliche Lage Symptome einer gespaltenen Konjunktur auf - die Exportwirtschaft boomt, die Binnennachfrage lahmt; allerdings steigen jetzt endlich die Investitionen, wozu auch der aufgestaute Bedarf an Ersatzinvestitionen beiträgt. Eine längerfristig anhaltende Wachstumsphase - und diese wird beschäftigungs- und sozialpolitisch dringend in Deutschland gebraucht - benötigt zwingend ein mitlaufendes Wachstum des pri- vaten Verbrauchs, der immerhin nahezu $60 \%$ der Gesamtnachfrage ausmacht. Da die Geldpolitik der Europäischen Zentralbank (EZB) zunehmend die wirtschaftliche Entwicklung durch Zinssteigerungen abbremst und auch die deutsche Fiskalpolitik deutlich restriktiv ausgerichtet ist, rechnen viele Prognosen mit einer Verminderung des Wachstumstempos 2007 um ca. 1 Prozentpunkt gegenüber dem Vorjahr - von 2,5 \% 2006 auf ca. 1,5 \% 2007. Jedoch könnte diese Verlangsamung durch kräftiges Wachstum der Löhne gedämpft werden. Dass dadurch die Exportwirtschaft ernsthaft geschwächt und die Investitionsdynamik gebrochen wird, ist ebenso unwahrscheinlich wie eine lohnbedingte Inflationsbeschleunigung, wenn die Lohnpolitik vernünftigen gesamtwirtschaftlichen Regeln folgt.

In Deutschland sind die Bruttolohnkosten je Stunde nominal in den Jahren 2000 bis 2006 um 1,3 \% jährlich gestiegen, die Gewinne und Vermögenseinkommen dagegen um 5,4\% (Tabelle 1). Die nominalen Lohnsteigerungen je Stunde haben seit 2000 nicht einmal den Anstieg der Verbraucherpreise - im Durchschnitt 1,6\% per annum - wettgemacht. Am Produktivitätsfortschritt waren Arbeitnehmereinkommen überhaupt nicht beteiligt. Dieser lag im Durchschnitt der Volkswirtschaft 2000-2006 bei 1,4 \% jährlich (gerechnet je Arbeitsstunde). Der durchschnittliche reale Bruttolohn je Arbeitnehmer lag 2006 um 4,6\% unter dem Wert von 1995. Zum Vergleich: In den USA stiegen 2000-2006 die nominalen Bruttolöhne je Arbeitnehmer um 4,0 \% jährlich, in Großbritannien um $4,5 \%$, auch die Reallöhne stiegen dabei deutlich. Dort waren Wirtschaftswachstum und Beschäftigung unvergleichlich kräftiger als hierzulande.

In der Metallindustrie stiegen die Stundenlöhne brutto mit ca. 2,2 \% im Zeitraum 2000-2006 zwar etwas stärker als im ge- samtwirtschaftlichen Durchschnitt, blieben aber auch hier noch etwas unter dem gesamtwirtschaftlichen Verteilungsspielraum. In der Metallindustrie ist der Produktivitätstrend in diesem Zeitraum mit etwa 3,8 \% 2,7-mal so hoch wie im Durchschnitt der Volkswirtschaft, sodass die Unternehmen dieser Branche von einer für sie außerordentlich günstigen Lohnstückkostenentwicklung profitieren konnten.

\section{VON DER LOHNDEFLATION ZURÜCK ZUR PRODUKTIVITÄTS- ORIENTIERTEN LOHNPOLITIK}

Die nominalen Lohnstückkosten - die Lohnkosten je Einheit Output (Bruttoinlandsprodukt) - sind in der Gesamtwirt-

Trevor Evans, Professor für Volkswirtschaftslehre an der FHW Berlin. Arbeitsgebiet: Internationale Wirtschaftsbeziehungen. e-mail: evans@fhw-berlin.de - Arne Heise, Professor für Volkswirtschaftslehre an der Universität Hamburg. Arbeitsgebiete: Wirtschaftspolitik, Makroökonomie, Europäische Integration.e-mail: arne.heise@wiso.unihamburg.de - Hansjörg Herr, Professor für Volkswirtschaftslehre an der FHW Berlin, Arbeitsgebiete: Makroökonomie, Geld- und Währungstheorie, Entwicklungsländer Jürgen Kromphardt, Professor em. für Volkswirtschaftslehre, TU Berlin, ehemaliges Mitglied im Sachverständigenrat. Arbeitsgebiete: Konjunktur, Wachstum, Beschäftigung. e-mail: J.Kromphardt@ww.tu-berlin.de Jan Priewe, Professor für Volkswirtschaftslehre an der FHTW Berlin. Arbeitsgebiete: Makroökonomie, Wirtschaftspolitik, Entwicklungsländer. e-mail: priewe@fhtw-berlin.deClaus Thomasberger, Professor für Volkswirtschaftslehre, FHTW Berlin. Arbeitsgebiete: Internationale Wirtschaftsbeziehun gen, Europäische Wirtschaft. e-mail: c.thomasberger@fhtw-berlin.de 


\begin{tabular}{|c|c|c|c|c|c|c|c|}
\hline & \multicolumn{2}{|c|}{ Arbeitnehmerentgelt je Stunde } & \multirow{2}{*}{$\begin{array}{l}\text { Unternehmens- } \\
\text { und Vermögens- } \\
\text { einkommen }\end{array}$} & \multirow{2}{*}{$\begin{array}{l}\text { Verbraucher- } \\
\text { Preise }\end{array}$} & \multicolumn{2}{|c|}{ Arbeitsproduktivität je Stunde } & \multirow{2}{*}{$\begin{array}{c}\text { Nominale } \\
\text { Lohnstückkosten } \\
\text { je Stunde, } \\
\text { Gesamtwirtschaft }\end{array}$} \\
\hline & $\begin{array}{l}\text { Gesamt- } \\
\text { wirtschaft }\end{array}$ & $\begin{array}{c}\text { Metall- } \\
\text { industrie (a) }\end{array}$ & & & $\begin{array}{c}\text { Gesamt- } \\
\text { wirtschaft }\end{array}$ & $\begin{array}{c}\text { Metall- } \\
\text { industrie }(a, c)\end{array}$ & \\
\hline 2000 & 3,3 & 2,3 & $-0,8$ & 1,4 & 2,6 & 8,5 & 0,7 \\
\hline 2001 & 2,5 & 3,8 & 3,7 & 2,0 & 1,8 & 2,1 & 0,6 \\
\hline 2002 & 2,0 & 3,6 & 1,7 & 1,4 & 1,5 & 1,9 & 0,6 \\
\hline 2003 & 2,0 & 1,7 & 3,9 & 1,1 & 1,2 & 2,4 & 0,7 \\
\hline 2004 & 0,1 & 0,0 & 10,4 & 1,6 & 0,7 & 4,5 & $-0,5$ \\
\hline 2005 & 0,4 & 1,5 & 6,2 & 2,0 & 1,3 & 5,3 & $-0,9$ \\
\hline 2006 & 0,7 & $2,7(b)$ & 6,9 & 1,7 & 1,9 & $6,9(b)$ & $-1,1$ \\
\hline $2000-2006(d)$ & 1,3 & 2,2 & 5,4 & 1,6 & 1,4 & $3,8(c)$ & 0,0 \\
\hline \multicolumn{7}{|c|}{$\begin{array}{l}\text { (a) Angaben von Gesamtmetall. (b) Angabe IG Metall Tarifabteilung. (c) Steigerungsraten ab } 2001 \text { wegen statistischer Umstellungen nicht vollständig vergleichbar. } \\
\text { (d) Durchschnittliche Veränderungsrate gegenüber dem Vorjahr 2001-2006. } \\
\text { Quelle: Statistisches Bundesamt; Gesamtmetall Homepage zu Angaben Metallindustrie; Berechnungen der Autoren. }\end{array}$} & $\begin{array}{l}\text { Hans Böckler } \\
\text { Stiftung }\end{array}$ \\
\hline
\end{tabular}

\begin{tabular}{|c|c|c|c|c|c|c|}
\hline $\begin{array}{l}\text { Nominale Lohnstückkosten- } \\
\text { entwicklung, Index } 2000=100\end{array}$ & 2001 & 2002 & 2003 & 2004 & 2005 & 2006 \\
\hline Euro-Zone & 102,3 & 104,7 & 106,8 & 107,8 & 108,9 & 109,8 \\
\hline Deutschland & 100,9 & 101,8 & 102,7 & 102,5 & 101,6 & 100,0 \\
\hline Spanien & 103,2 & 106,2 & 109,3 & 112,0 & 114,5 & 117,4 \\
\hline Frankreich & 102,3 & 105,7 & 107,1 & 108,2 & 110,2 & 112,3 \\
\hline Italien & 103,2 & 106,9 & 111,6 & 114,3 & 117,1 & 120,1 \\
\hline GB & 103,5 & 105,6 & 108,8 & 111,1 & 115,2 & 118,7 \\
\hline Tschechien & 105,8 & 112,1 & 116,2 & 118,4 & 118,1 & 118,6 \\
\hline USA & 101,9 & 102,7 & 104,4 & 105,6 & 107,5 & 111,9 \\
\hline Japan & 98,4 & 95,2 & 91,9 & 88,5 & 87,2 & 85,9 \\
\hline Quelle: AMECO-Datenbank Eurostat. & & & & \multicolumn{3}{|c|}{ WSI $\begin{array}{c}\text { Hans Böckle } \\
\text { Stiftung }\end{array}$} \\
\hline
\end{tabular}

schaft seit 2000 unverändert geblieben, in den letzten Jahren sind sie sogar etwas gesunken. Dies war eine deflationäre Lohnentwicklung: Wenn nur die Lohnkosten die Preisniveauentwicklung bestimmt hätten, hätten wir Deflation in Deutschland gehabt. Im Vergleich zu allen anderen Handelspartnerländern hat nur Japan eine noch schwächere Lohnstückkostenentwicklung seit 2000 verzeichnet (Tabelle 2). Mit sehr niedrigen Lohnstückkosten hat sich die deutsche Wirtschaft in der Eurozone, aber auch gegenüber Großbritannien und den USA, den Weltmeistertitel im Export - und zugleich hohe Gewinne auf dem Rücken der Arbeitnehmer - geholt, unterstützt von der guten Konjunktur in den USA und in der Weltwirtschaft, die zu einer starken Nachfrage nach deutschen Investitionsgütern geführt hat. Der Preis für die deutsche Lohnzurückhaltung ist hoch: steigende Handelsbilanzungleichgewichte in der EU, aber auch in den USA und bei anderen Handelspartnerländern, zugleich Schwäche der Binnennachfrage in Deutschland und zunehmende Verteilungsunge-

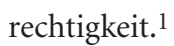

Die deutsche Lohnentwicklung der vergangenen Jahre war Ausdruck einer typi- schen „beggar my neighbour“-Politik: Deutschland hat mit niedrigen Lohnstückkosten die europäischen Nachbarländer, insbesondere jene in der Euro-Zone, unterboten und dadurch Arbeitslosigkeit exportiert. 2006 lag der deutsche Exportüberschuss (bei Gütern und Dienstleistungen) bei 5,0 \% des BIP. Ein solch hoher Überschuss, der zu einem großen Teil gegenüber Ländern in der Euro-Zone entstand, muss zwangsläufig zu schweren Spannungen in der Europäischen Währungsunion führen. Denn hier sind Wechselkursanpassungen zum Ausgleich der Ungleichgewichte nicht mehr möglich. Daher muss die Lohnpolitik dazu beitragen, dass hohe Handelsbilanzüberschüsse (und Defizite bei Handelspartnern) gar nicht erst entstehen bzw. langsam wieder zurückgeführt werden. Eine Fortsetzung der deutschen Lohnzurückhaltung der vergangenen Jahre würde die Spannungen weiter verschärfen.

Wir plädieren für eine Verstetigung der Lohnentwicklung durch eine Tariflohnpolitik, die sich - im Grundsatz - an der gesamtwirtschaftlichen Produktivitätssteigerung und der von der EZB festgelegten Zielinflation von 2,0\% orientiert. Wer al- lerdings der Abkehr vom Flächentarifvertrag und der Lohnflexibilisierung das Wort redet, müsste jetzt prozyklisch für sehr hohe Lohnforderungen, die sich an den Gewinnsteigerungen orientieren, und in schwachen Jahren für sehr niedrige Steigerungen oder gar Lohnkürzungen plädieren. Dies halten wir für konjunkturund verteilungspolitisch schädlich. Da die trendmäßige Produktivitätssteigerung (Arbeitsproduktivität je Stunde 2000-2006) bei $1,4 \%$ (schwankend zwischen $0,7 \%$ und $1,9 \%$ seit 2001) liegt, wäre eine realisierte Zunahme aller Arbeitsentgelte in allen Branchen um rund 3,5 \% vernünftig. Wer meint, dass der Produktivitätstrend verzerrt sei durch „entlassungsbedingte Produktivitätssteigerungen“, müsste auch andere, entgegenwirkende Verzerrungen einbeziehen, wie zum Beispiel eine durch schlechte Kapazitätsauslastung verursachte schwache Produktivitätsdynamik. Wir halten derartige Bereinigungen für problematisch und beziehen uns daher auf den mittelfristigen Produktivitätstrend.

\footnotetext{
Manchmal wird darauf verwiesen, dass das deutsche Lohnstückkostenniveau überhöht sei und daher unterdurchschnittliche Zuwächse gegenüber anderen Ländern geboten seien. Wer so argumentiert, nimmt die Fakten nicht zur Kenntnis: Hierzulande lagen 2006 die durchschnittlichen Arbeitsentgelte je Beschäftigten bei $41.460 €$, in den Niederlanden erreichten sie inzwischen $45.500 €$, in Großbritannien $42.400 €$ (mit aktuellem Wechselkurs umgerechnet), in Irland $42.420 €$, in den USA sogar $46.940 €$ (mit aktuellem Wechselkurs umgerechnet). Frankreichs Löhne haben mit den deutschen nahezu gleichgezogen, ausgehend von einem deutlich niedrigerem Niveau (nach Eurostat, AMECO-Datenbank). Angaben über die Arbeitnehmereinkommen je Stunde im internationalen Vergleich liegen leider nicht vor.
} 


\section{GEFÄHRDUNG DER KONJUNKTUR DURCH ZU HOHE LÖHNE?}

Manche Kommentatoren warnen vor $\mathrm{zu}$ starken Lohnsteigerungen angesichts einer noch labilen Konjunktur, weil sie Exporte und Investitionen gefährden würden. Dabei wird übersehen, dass Lohnsteigerungen mit hoher Wahrscheinlichkeit zu konjunkturstabilisierendem Konsumwachstum führen, dass eine forcierte Exportüberschusspolitik nicht wünschenswert ist und dass angesichts der starken Gewinnentwicklung ein Einbruch der Investitionstätigkeit nicht zu befürchten ist; jedenfalls dann nicht, wenn eine produktivitäts- und damit kostenneutrale Lohnpolitik angestrebt wird und die Geldpolitik nicht restriktiv übersteuert. Wer kräftige Lohnsteigerungen in der jetzigen Phase für fragwürdig hält, muss sich zudem fragen lassen: Wann denn dann?

In der Metallindustrie, die etwa die Hälfte der Arbeitsplätze in der Industrie repräsentiert, liegen die Produktivitätssteigerungen deutlich über dem erwähnten gesamtwirtschaftlichen Durchschnitt. Die Gewinnsteigerungen sind kräftiger als im Durchschnitt der Volkswirtschaft. Alle, die eine Lohnentwicklung propagieren, die sich an der Branchenproduktivität und den Branchengewinnen orientiert, müssten nun deutlich höhere Lohnsteigerungen gutheißen. Wir lehnen eine solche Orien- tierung ab, weil sie zu einer unausgewogenen, nicht an Qualifikation und Leistung ausgerichteten sektoralen Lohnentwicklung führt. Wenn jedoch - wie zu befürchten - die Lohnentwicklung in den Dienstleistungssektoren wie in den vergangenen Jahren besonders schwach bleibt, weil hier auch die Produktivitätsentwicklung hinter dem gesamtwirtschaftlichen Produktivitätstrend zurückbleibt, dann halten wir es für vertretbar, ja wünschenswert, wenn die Lohnentwicklung in der Metallindustrie die Lohnschwäche in den anderen Sektoren teilweise kompensiert. Ein solcher Tarifabschluss in der Metallindustrie, der dann mehr als 3,5 \% Zuwachs je Stunde bringen würde, wäre sehr wohl mit gesamtwirtschaftlicher Stabilität vereinbar. Was die angemessenen Forderungen sind, um dieses Ziel zu erreichen, steht auf einem anderen Blatt, weil sie auch von taktischen Erwägungen abhängen.

Eine andere Variante besteht darin, zusätzlich zur produktivitätsorientierten Lohnpolitik im Flächentarifvertrag unternehmensspezifische Einmalzahlungen (Konjunkturbonus, bezogen auf die Metallindustrie) zu vereinbaren, die der jeweiligen Gewinnsituation Rechnung tragen. Die vor allem von manchen Arbeitgebern favorisierten Einmalzahlungen sind jedoch kein Ersatz für produktivitätsorientierte Lohnzuwächse, die dauerhaft Bestand haben sollten, weil weder die Produktivitäts- steigerung noch die Inflation vorübergehende Einmaleffekte sind.

Einige Vertreter der Deutschen Bundesbank und der EZB haben Befürchtungen geäußert, dass zu kräftige Lohnsteigerungen in der deutschen Metallindustrie zu einer weiteren Straffung der Geldpolitik führen könnten, weil eine europäische Lohn-Preis-Spirale befürchtet wird. Wir teilen diese Befürchtungen nicht, denn es gibt nicht die geringsten Anhaltspunkte für eine bevorstehende „Lohnorgie“ in der Eurozone. Die Geldpolitik sollte sich nicht von spekulativen Ängsten leiten lassen. Allerdings darf die leicht unterdurchschnittliche deutsche Inflationsrate der vergangenen Jahre - die vor allem eine Folge der Lohnzurückhaltung ist - nicht als Legitimation für über der Zielinflationsrate liegende Preissteigerungen in einigen anderen Ländern der Eurozone herhalten. Auch aus der Sicht der europäischen Geldpolitik müsste klar sein, dass jedes Mitgliedsland der Währungsunion seinen Verteilungsspielraum ausnutzen darf und auch ausnutzen sollte. Nicht legitim sind Zinsdrohungen gegen jene, die sich stabilitätsgerecht verhalten. Die von uns geforderten Lohnsteigerungen in der Metallindustrie von über $4 \%$ verlassen nicht den Rahmen einer gesamtwirtschaftlichen Lohnentwicklung, die mit der von der EZB gewählten Zielinflationsrate von $2 \%$ vereinbar ist. 